\title{
On reference frames in spacetime and gravitational energy in freely falling frames
}

\author{
J. W. Maluf*, F. F. Faria and S. C. Ulhoa \\ Instituto de Física, \\ Universidade de Brasília \\ C. P. 04385 \\ 70.919-970 Brasília DF, Brazil
}

\begin{abstract}
We consider the interpretation of tetrad fields as reference frames in spacetime. Reference frames may be characterized by an antisymmetric acceleration tensor, whose components are identified as the inertial accelerations of the frame (the translational acceleration and the frequency of rotation of the frame). This tensor is closely related to gravitoelectromagnetic field quantities. We construct the set of tetrad fields adapted to observers that are in free fall in the Schwarzschild spacetime, and show that the gravitational energy-momentum constructed out of this set of tetrad fields, in the framework of the teleparallel equivalent of general relatrivity, vanishes. This result is in agreement with the principle of equivalence, and may be taken as a condition for a viable definition of gravitational energy.
\end{abstract}

PACS numbers: 04.20.Cv, 04.20.Fy

(*) e-mail: wadih@unb.br 


\section{Introduction}

It is a long-established practice in physics to describe the gravitational field by means of theories invariant under local Lorentz transformations. This is the case of the Einstein-Cartan theory, for instance, or more generally of the metric-affine approach to the gravitational field [1]. In the latter formulation, the theory of gravity is considered as a gauge theory of the Poincaré group. The motivation for addressing theories of gravity by means of local Lorentz $(\mathrm{SO}(3,1))$ symmetry is partially due to the impact of the Yang-Mills gauge theory in particle physics and quantum field theory. Because of the local $\mathrm{SO}(3,1)$ symmetry, it is possible to assert that in such theories "all reference frames are equivalent".

The investigation of metric-affine theories of gravity is important because one might have to go beyond the Riemannian formulation of general relativity in order to deal with structures that pertain to a possible quantum theory of gravity. The relevance of the Poincaré group and its representations in quantum field theory is well known. In spite of the above mentioned feature of the local $\mathrm{SO}(3,1)$ symmetry, there is no physical reason that prevents the possibility of considering theories of gravity invariant under the global Lorentz symmetry.

One theory that exhibits invariance under global $\mathrm{SO}(3,1)$ symmetry is the teleparallel equivalent of general relativity (TEGR) $[2,3,4,5,6,7,8]$. The Lagrangian density of the theory is invariant under local $\mathrm{SO}(3,1)$ transformations up to a nontrivial, nonvanishing total divergence [9], and for this reason the local $\mathrm{SO}(3,1)$ group is not a symmetry of the theory. (From a different perspective, the TEGR may be considered as a gauge theory for the translation group [10].) Because of the global $\mathrm{SO}(3,1)$ symmetry, we must ascribe an interpretation to six degrees of freedom of the tetrad field. In the TEGR two sets of tetrad fields that yield the same spacetime metric tensor are physically distinct. Thus we should interpret the tetrad fields as reference frames adapted to ideal observers in spacetime. Therefore two sets of tetrad fields that are related by a local $\mathrm{SO}(3,1)$ transformation yield the same metrical properties of the spacetime, but represent reference frames that are characterized by different inertial accelerations. In a given gravitational field configuration, the Schwarzschild spacetime, say, a moving observer or an observer at rest are described by different sets of tetrad fields, and both sets of tetrads are related by some sort of $\mathrm{SO}(3,1)$ transformation. Of course the 
proper interpretation of the translational and rotational accelerations of a frame makes sense at least in the case of asymptotically flat spacetimes.

In this paper we carry out an analysis of the inertial accelerations of a frame in the context of the TEGR. The inertial accelerations are represented by a second rank antisymmetric tensor under global $\mathrm{SO}(3,1)$ transformations that is coordinate independent. This tensor can be decomposed into translational and rotational accelerations (the latter is in fact the rotational frequency of the frame). By considering the weak field limit we will see that there is a very interesting relationship between the translational acceleration and rotational frequency of the frame, and electric and magnetic fields, respectively. This relationship is explicitly investigated in the context of the Kerr spacetime. The translational acceleration and rotational frequency that are necessary no maintain a static frame in the spacetime are closely related to the electric field of a point charge and to the magnetic field of a perfect magnetic dipole, respectively. The present analysis is very much similar to the usual formulation of gravitoelectromagnetism.

We consider the four-velocity of observers that are in free fall (radially) in the Schwarzschild spacetime and construct the reference frame adapted to such observers. We show that the expression for the gravitational energymomentum that arises in the framework of the TEGR [4, 5, 7] vanishes, if evaluated in this frame. This is a very interesting result that shows the consistency of the above definition with the principle of equivalence. The local effects of gravity are not measured by an observer in free fall, who defines a locally inertial reference frame. In this frame the acceleration of the observer vanishes (section 3), and therefore he cannot measure neither the gravitational force exerted on him nor the mass of the black hole. Thus in a freely falling frame the gravitational energy should vanish. The tetrad field that establishes the reference frame of an observer in free fall is related to other (possibly static) frames by a frame transformation, not a coordinate transformation. For instance, it is possible to establish a transformation from the freely falling frame to a frame adapted to observers that are asympotically at rest in the Schwarzschild spacetime, out of which we obtain the usual value for the total gravitational energy of the spacetime. We believe that viable definitions of gravitational energy-momentum should exhibit this feature.

Notation: spacetime indices $\mu, \nu, \ldots$ and $\mathrm{SO}(3,1)$ indices $a, b, \ldots$ run from 0 to 3 . Time and space indices are indicated according to $\mu=0, i, \quad a=$ 
$(0),(i)$. The tetrad field is denoted by $e^{a}{ }_{\mu}$, and the torsion tensor reads $T_{a \mu \nu}=\partial_{\mu} e_{a \nu}-\partial_{\nu} e_{a \mu}$. The flat, Minkowski spacetime metric tensor raises and lowers tetrad indices and is fixed by $\eta_{a b}=e_{a \mu} e_{b \nu} g^{\mu \nu}=(-+++)$. The determinant of the tetrad field is represented by $e=\operatorname{det}\left(e^{a}{ }_{\mu}\right)$.

\section{The field equations of the TEGR}

Einstein's general relativity is determined by the field equations. The latter may be written either in terms of the metric tensor or of the tetrad field. The TEGR is a reformulation of Einstein's general relativity in terms of the tetrad field. Sometimes the theory is also called "tetrad gravity" [9]. The tetrad field is anyway necessary to describe the coupling of Dirac spinor fields with the gravitational field. The formulation of general relativity in a different geometrical framework allows a new insight into the theory, and this is precisely what happens in the consideration of the TEGR.

The Lagrangian density for the gravitational field in the TEGR is given by

$$
\begin{aligned}
L & =-k e\left(\frac{1}{4} T^{a b c} T_{a b c}+\frac{1}{2} T^{a b c} T_{b a c}-T^{a} T_{a}\right)-L_{M} \\
& \equiv-k e \Sigma^{a b c} T_{a b c}-L_{M}
\end{aligned}
$$

where $k=1 /(16 \pi)$, and $L_{M}$ stands for the Lagrangian density for the matter fields. As usual, tetrad fields convert spacetime into Lorentz indices and viceversa. The tensor $\Sigma^{a b c}$ is defined by

$$
\Sigma^{a b c}=\frac{1}{4}\left(T^{a b c}+T^{b a c}-T^{c a b}\right)+\frac{1}{2}\left(\eta^{a c} T^{b}-\eta^{a b} T^{c}\right),
$$

and $T^{a}=T^{b}{ }_{b}{ }^{a}$. The quadratic combination $\Sigma^{a b c} T_{a b c}$ is proportional to the scalar curvature $R(e)$, except for a total divergence [7].

The field equations for the tetrad field read

$$
e_{a \lambda} e_{b \mu} \partial_{\nu}\left(e \Sigma^{b \lambda \nu}\right)-e\left(\Sigma_{a}^{b \nu} T_{b \nu \mu}-\frac{1}{4} e_{a \mu} T_{b c d} \Sigma^{b c d}\right)=\frac{1}{4 k} e T_{a \mu} .
$$


where $e T_{a \mu}=\delta L_{M} / \delta e^{a \mu}$. It is possible to prove by explicit calculations that the left hand side of Eq. (3) is exactly given by $\frac{1}{2} e\left[R_{a \mu}(e)-\frac{1}{2} e_{a \mu} R(e)\right]$. The field equations above may be rewritten in the form

$$
\partial_{\nu}\left(e \Sigma^{a \lambda \nu}\right)=\frac{1}{4 k} e e_{\mu}^{a}\left(t^{\lambda \mu}+T^{\lambda \mu}\right),
$$

where

$$
t^{\lambda \mu}=k\left(4 \Sigma^{b c \lambda} T_{b c}{ }^{\mu}-g^{\lambda \mu} \Sigma^{b c d} T_{b c d}\right),
$$

is interpreted as the gravitational energy-momentum tensor [7].

The Lagrangian density defined by Eq. (1) is invariant under global $\mathrm{SO}(3,1)$ transformations of the tetrad field. As we asserted before, under local $\mathrm{SO}(3,1)$ transformations the purely gravitational part of Eq. (1), $-k e \sum^{a b c} T_{a b c}$, transforms into $-k e \sum^{a b c} T_{a b c}$ plus a nontrivial, nonvanishing total divergence [9]. The integral of this total divergence in general is nonvanishing, unless restrictive conditions are imposed on the Lorentz transformation matrices.

The Hamiltonian formulation of the TEGR is obtained by first establishing the phase space variables. The Lagrangian density does not contain the time derivative of the tetrad component $e_{a 0}$. Therefore this quantity will arise as a Lagrange multiplier. The momentum canonically conjugated to $e_{a i}$ is given by $\Pi^{a i}=\delta L / \delta \dot{e}_{a i}$. The Hamiltonian formulation is obtained by rewriting the Lagrangian density in the form $L=p \dot{q}-H$, in terms of $e_{a i}$, $\Pi^{a i}$ and Lagrange multipliers. The Legendre transform can be successfuly carried out, and the final form of the Hamiltonian density reads [11]

$$
H=e_{a 0} C^{a}+\alpha_{i k} \Gamma^{i k}+\beta_{k} \Gamma^{k},
$$

plus a surface term. $\alpha_{i k}$ and $\beta_{k}$ are Lagrange multipliers that (after solving the field equations) are identified as $\alpha_{i k}=1 / 2\left(T_{i 0 k}+T_{k 0 i}\right)$ and $\beta_{k}=T_{00 k}$. $C^{a}, \Gamma^{i k}$ and $\Gamma^{k}$ are first class constraints.

The constraint $C^{a}$ is written as $C^{a}=-\partial_{i} \Pi^{a i}+h^{a}$, where $h^{a}$ is an intricate expression of the field variables. The integral form of the constraint equation $C^{a}=0$ motivates the definition of the total energy-momentum four-vector $P^{a}[4]$,

$$
P^{a}=-\int_{V} d^{3} x \partial_{i} \Pi^{a i} .
$$


$V$ is an arbitrary volume of the three-dimensional space. In the configuration space we have

$$
\Pi^{a i}=-4 k e \Sigma^{a 0 i}
$$

The emergence of total divergences in the form of scalar or vector densities is possible in the framework of theories constructed out of the torsion tensor. Metric theories of gravity do not share this feature. We note that by making $\lambda=0$ in eq. (4) and identifying $\Pi^{a i}$ in the left hand side of the latter, the integral form of eq. (4) is written as

$$
P^{a}=\int_{V} d^{3} x e e^{a}{ }_{\mu}\left(t^{0 \mu}+T^{0 \mu}\right) .
$$

In empty spacetimes and in the framework of black holes $P^{a}$ does represent the gravitational energy-momentum contained in a volume $V$ of the threedimensional space. Several applications to well known gravitational field configurations support this interpretation.

\section{Reference frames in spacetime}

A set of four orthonormal, linearly independent vector fields in spacetime establish a reference frame. Altogether, they define a tetrad field $e^{a}{ }_{\mu}$, which allows the projection of vectors and tensors in spacetime in the local frame of an observer.

Each set of tetrad fields defines a class of reference frames [12]. If we denote by $x^{\mu}(s)$ the world line $C$ of an observer in spacetime ( $s$ is the proper time of the observer), and by $u^{\mu}(s)=d x^{\mu} / d s$ its velocity along $C$, we identify the observer's velocity with the $a=(0)$ component of $e_{a}{ }^{\mu}$. Thus $u^{\mu}(s)=$ $e_{(0)}{ }^{\mu}$ along $C$. The acceleration $a^{\mu}$ of the observer is given by the absolute derivative of $u^{\mu}$ along $C$,

$$
a^{\mu}=\frac{D u^{\mu}}{d s}=\frac{D e_{(0)}^{\mu}}{d s}=u^{\alpha} \nabla_{\alpha} e_{(0)}^{\mu},
$$

where the covariant derivative is constructed out of the Christoffel symbols. Thus $e_{a}{ }^{\mu}$ determines the velocity and acceleration along the worldline of an observer adapted to the frame. Therefore a given set of tetrad fields, for which $e_{(0)}^{\mu}$ describes a congruence of timelike curves, is adapted to a particular 
class of observers, namely, to observers characterized by the velocity field $u^{\mu}=e_{(0)}{ }^{\mu}$, endowed with acceleration $a^{\mu}$. If $e^{a}{ }_{\mu} \rightarrow \delta_{\mu}^{a}$ in the limit $r \rightarrow \infty$, then $e^{a}{ }_{\mu}$ is adapted to static observers at spacelike infinity.

A geometrical characterization of tetrad fields as an observer's frame can be given by considering the acceleration of the frame along an arbitrary path $x^{\mu}(s)$ of the observer in spacetime. The acceleration of the frame is determined by the absolute derivative of $e_{a}^{\mu}$ along $x^{\mu}(s)$. Thus, assuming that the observer carries an orthonormal tetrad frame $e_{a}{ }^{\mu}$, the acceleration of the latter along the path is given by $[13,14]$

$$
\frac{D e_{a}{ }^{\mu}}{d s}=\phi_{a}{ }^{b} e_{b}{ }^{\mu}
$$

where $\phi_{a b}$ is the antisymmetric acceleration tensor. According to Refs. [13, $14]$, in analogy with the Faraday tensor we can identify $\phi_{a b} \rightarrow(\mathbf{a}, \boldsymbol{\Omega})$, where $\mathbf{a}$ is the translational acceleration $\left(\phi_{(0)(i)}=a_{(i)}\right)$ and $\boldsymbol{\Omega}$ is the frequency of rotation of the local spatial frame with respect to a nonrotating (FermiWalker transported [12]) frame. It follows from Eq. (11) that

$$
\phi_{a}{ }^{b}=e^{b}{ }_{\mu} \frac{D e_{a}^{\mu}}{d s}=e^{b}{ }_{\mu} u^{\lambda} \nabla_{\lambda} e_{a}^{\mu} .
$$

Therefore given any set of tetrad fields for an arbitrary gravitational field configuration, its geometrical interpretation can be obtained by suitably interpreting the velocity field $u^{\mu}=e_{(0)}^{\mu}$ and the acceleration tensor $\phi_{a b}$. The acceleration vector $a^{\mu}$ defined by Eq. (10) may be projected on a frame in order to yield

$$
a^{b}=e^{b}{ }_{\mu} a^{\mu}=e^{b}{ }_{\mu} u^{\alpha} \nabla_{\alpha} e_{(0)}{ }^{\mu}=\phi_{(0)}{ }^{b} .
$$

Thus $a^{\mu}$ and $\phi_{(0)(i)}$ are not different accelerations of the frame.

The expression of $a^{\mu}$ given by Eq. (10) may be rewritten as

$$
\begin{aligned}
a^{\mu} & =u^{\alpha} \nabla_{\alpha} e_{(0)}^{\mu}=u^{\alpha} \nabla_{\alpha} u^{\mu}=\frac{d x^{\alpha}}{d s}\left(\frac{\partial u^{\mu}}{\partial x^{\alpha}}+\Gamma_{\alpha \beta}^{\mu} u^{\beta}\right) \\
& =\frac{d^{2} x^{\mu}}{d s^{2}}+\Gamma_{\alpha \beta}^{\mu} \frac{d x^{\alpha}}{d s} \frac{d x^{\beta}}{d s},
\end{aligned}
$$

where $\Gamma_{\alpha \beta}^{\mu}$ are the Christoffel symbols. We see that if $u^{\mu}=e_{(0)}{ }^{\mu}$ represents a geodesic trajectory, then the frame is in free fall and $a^{\mu}=\phi_{(0)(i)}=0$. 
Therefore we conclude that nonvanishing values of the latter quantities do represent inertial accelerations of the frame.

In view of the orthogonality of the tetrads we write Eq. (12) as $\phi_{a}{ }^{b}=$ $-u^{\lambda} e_{a}{ }^{\mu} \nabla_{\lambda} e^{b}{ }_{\mu}$, where $\nabla_{\lambda} e^{b}{ }_{\mu}=\partial_{\lambda} e^{b}{ }_{\mu}-\Gamma_{\lambda \mu}^{\sigma} e^{b}{ }_{\sigma}$. Now we take into account the identity $\partial_{\lambda} e^{b}{ }_{\mu}-\Gamma_{\lambda \mu}^{\sigma} e^{b}{ }_{\sigma}+{ }^{0} \omega_{\lambda}{ }^{b}{ }_{c} e^{c}{ }_{\mu}=0$, where ${ }^{0} \omega_{\lambda}{ }^{b}{ }_{c}$ is the metric compatible, torsion free Levi-Civita connection, and express $\phi_{a}{ }^{b}$ according to

$$
\phi_{a}^{b}=e_{(0)}^{\mu}\left({ }^{0} \omega_{\mu}^{b}{ }_{a}\right) .
$$

At last we consider the identity ${ }^{0} \omega_{\mu}{ }^{a}{ }_{b}=-K_{\mu}{ }^{a}{ }_{b}$, where $-K_{\mu}{ }^{a}{ }_{b}$ is the contortion tensor defined by

$$
K_{\mu a b}=\frac{1}{2} e_{a}^{\lambda} e_{b}^{\nu}\left(T_{\lambda \mu \nu}+T_{\nu \lambda \mu}+T_{\mu \lambda \nu}\right),
$$

and $T_{\lambda \mu \nu}=e^{a}{ }_{\lambda} T_{a \mu \nu}$ (see, for instance, Eq. (4) of Ref. [7]; the identity is obtained by requiring the vanishing of a general $\mathrm{SO}(3,1)$ connection $\omega_{\mu a b}$, or by direct calculation). After simple manipulations we finally obtain

$$
\phi_{a b}=\frac{1}{2}\left[T_{(0) a b}+T_{a(0) b}-T_{b(0) a}\right] .
$$

The expression above is clearly not invariant under local $\mathrm{SO}(3,1)$ transformations, but is invariant under coordinate transformations. The values of $\phi_{a b}$ for a given tetrad field may be used to characterize the frame. We recall that we are assuming the observer to carry the set of tetrad fields along $x^{\mu}(s)$, for which we have $u^{\mu}=e_{(0)}{ }^{\mu}$. We interpret $\phi_{a b}$ as the inertial accelerations along $x^{\mu}(s)$.

Two simple, straightforward applications of Eq. (17) are the following:

(i) The tetrad field adapted to observers at rest in Minkowski spacetime is given by $e^{a}{ }_{\mu}(c t, x, y, z)=\delta_{\mu}^{a}$. We consider a time-dependent boost in the $x$ direction, say, after which the tetrad field reads

$$
e_{\mu}^{a}(c t, x, y, z)=\left(\begin{array}{cccc}
\gamma & -\beta \gamma & 0 & 0 \\
-\beta \gamma & \gamma & 0 & 0 \\
0 & 0 & 1 & 0 \\
0 & 0 & 0 & 1
\end{array}\right)
$$


where $\gamma=\left(1-\beta^{2}\right)^{-1 / 2}, \beta=v / c$ and $v=v(t)$. The frame above is then adapted to observers whose four-velocity is $u^{\mu}=e_{(0)}^{\mu}(c t, x, y, z)=$ $(\gamma, \beta \gamma, 0,0)$. After simple calculations we obtain

$$
\begin{aligned}
& \phi_{(0)(1)}=\frac{d}{d x^{0}}[\beta \gamma]=\frac{d}{d t}\left[\frac{v / c^{2}}{\sqrt{1-v^{2} / c^{2}}}\right], \\
& \phi_{(0)(2)}=0 \\
& \phi_{(0)(3)}=0
\end{aligned}
$$

and $\phi_{(i)(j)}=0$.

(ii) A frame adapted to an observer in Minkowski spacetime whose fourvelocity is $e_{(0)}^{\mu}=(1,0,0,0)$ and which rotates around the $z$ axis, say, reads

$$
e_{\mu}^{a}(c t, x, y, z)=\left(\begin{array}{cccc}
1 & 0 & 0 & 0 \\
0 & \cos \omega(t) & -\sin \omega(t) & 0 \\
0 & \sin \omega(t) & \cos \omega(t) & 0 \\
0 & 0 & 0 & 1
\end{array}\right) .
$$

It is easy to carry out the simple calculations and obtain

$$
\begin{aligned}
& \phi_{(2)(3)}=0 \\
& \phi_{(3)(1)}=0 \\
& \phi_{(1)(2)}=-\frac{d \omega}{d x^{0}}
\end{aligned}
$$

and $\phi_{(0)(i)}=0$. Together with the discussion regarding Eq. (14), the examples above support the interpretation of $\phi_{a}{ }^{b}$ as the inertial accelerations of the frame.

\section{A freely falling frame in the Schwarzschild spacetime}

We will consider in this section a frame that is in free fall in the Schwarzschild spacetime, namely, that is radially accelerated towards the center of the black 
hole. We will take into account the kinematical quantities discussed the preceeding section, in order to illustrate the construction of the tetrad field.

The spacetime is described by the line element

$$
d s^{2}=-\alpha^{-2} d t^{2}+\alpha^{2} d r^{2}+r^{2}\left(d \theta^{2}+\sin ^{2} \theta d \phi^{2}\right)
$$

where

$$
\alpha^{-2}=1-\frac{2 m}{r} .
$$

Let us define the quantity $\beta$,

$$
\beta=\left(\frac{2 m}{r}\right)^{1 / 2}=\left(1-\alpha^{-2}\right)^{1 / 2},
$$

which will be useful in the following.

An observer that is in radial free fall in the Schwarzschild spacetime is endowed with the four-velocity [15]

$$
u^{\alpha}=\left[\left(1-\frac{2 m}{r}\right)^{-1},-\left(\frac{2 m}{r}\right)^{1 / 2}, 0,0\right] .
$$

The simplest set of tetrad fields that satisfies the condition

$$
e_{(0)}^{\alpha}=u^{\alpha}
$$

is given by

$$
e_{a \mu}=\left(\begin{array}{cccc}
-1 & -\alpha^{2} \beta & 0 & 0 \\
\beta \sin \theta \cos \phi & \alpha^{2} \sin \theta \cos \phi & r \cos \theta \cos \phi & -r \sin \theta \sin \phi \\
\beta \sin \theta \sin \phi & \alpha^{2} \sin \theta \sin \phi & r \cos \theta \sin \phi & r \sin \theta \cos \phi \\
\beta \cos \theta & \alpha^{2} \cos \theta & -r \sin \theta & 0
\end{array}\right)
$$

We recall that the index $a$ labels the lines, and $\mu$ the columns. Since the frame is in free fall the equation $\phi_{(0)(i)}=0$ is satisfied. It is not difficult to show that this set of tetrad fields also satisfies the conditions

$$
\phi_{(i)(j)}=\frac{1}{2}\left[T_{(0)(i)(j)}+T_{(i)(0)(j)}-T_{(j)(0)(i)}\right]=0 .
$$

Three of the four conditions established by Eq. (26) are more relevant for our purposes, namely, the three components of the frame velocity in 
the three-dimensional space, $u^{i}=e_{(0)}{ }^{i}$. Together with the three conditions determined by Eq. (28), we have six conditions on the frame. We may assert that these six conditions completely fix the structure of the tetrad field, even though Eq. (28) has been verified a posteriori. Therefore Eq. (27) describes a nonrotating frame in radial free fall in the Schwarzschild spacetime.

We will evaluate the gravitational energy-momentum out of the tetrad field above, but will omit the details of the calculations which are algebraically long, but otherwise simple. The nonvanishing components of the torsion tensor are

$$
\begin{aligned}
& T_{001}=-\beta \partial_{r} \beta \\
& T_{101}=-\alpha^{2} \partial_{r} \beta \\
& T_{202}=-r \beta \\
& T_{303}=-r \beta \sin ^{2} \theta \\
& T_{212}=r\left(1-\alpha^{2}\right) \\
& T_{313}=r\left(1-\alpha^{2}\right) \sin ^{2} \theta .
\end{aligned}
$$

The gravitational energy contained within a spherical surface of constant radius is given by

$$
P^{(0)}=-\oint_{S} d S_{j} \Pi^{(0) j}=4 k \oint_{S} d S_{1} e\left(e^{(0)}{ }_{0} \Sigma^{001}+e^{(0)}{ }_{1} \Sigma^{101}\right)
$$

where

$$
\begin{aligned}
\Sigma^{001} & =\frac{1}{2}\left(g^{00} g^{11} g^{22} T_{212}+g^{00} g^{11} g^{33} T_{313}\right) \\
\Sigma^{101} & =-\frac{1}{2}\left(g^{00} g^{11} g^{22} T_{202}+g^{00} g^{11} g^{33} T_{303}\right) .
\end{aligned}
$$

We find that

$$
\begin{aligned}
e\left(e^{(0)}{ }_{0} \Sigma^{001}+e^{(0)}{ }_{1} \Sigma^{101}\right) & =r \sin \theta\left(\alpha^{2}-1-\alpha^{2} \beta^{2}\right) \\
& =0
\end{aligned}
$$

and therefore the gravitational energy contained within a surface of constant radius as well as the total gravitational energy of the spacetime vanishes, if 
evaluated in the frame of a freely falling observer. This is a very interesting property of the whole formalism described in section 2 . The vanishing of the gravitational energy for freely falling observers is a feature that is consistent with (and a consequence of) the principle of equivalence, since local effects of gravity are not measured by observers in free fall. For other frames that are related to Eq. (27) by a local Lorentz transformation we obtain nonvanishing values of $P^{(0)}$. In particular, the total gravitational energy calculated out of frames such that $e^{a}{ }_{\mu}(t, x, y, z) \rightarrow \delta_{\mu}^{a}$ in the asymptotic limit $r \rightarrow \infty$ is exactly $P^{(0)}=m$ [4]. The latter tetrad field is adapted to observers at rest at spacelike infinity. Thus the vanishing of gravitational energy in freely falling frames shows that the localizability of the gravitational energy is not inconsistent with with the principle of equivalence. The result given by Eqs. (30-32) is a very good example of the frame dependence of the gravitational energy definition (7).

It can be easily verified that the gravitational momentum components

$P^{(1)}$ and $P^{(2)}$ vanish in view of integrals like $\int_{0}^{2 \pi} d \phi \sin \phi=0=\int_{0}^{2 \pi} d \phi \cos \phi$, whereas $P^{(3)}$ vanishes due to $\int_{0}^{\pi} d \theta \sin \theta \cos \theta=0$.

It is important to remark that in general the vanishing of $\phi_{a b}$ does not imply the vanishing of $P^{a}$. For an observer at rest at spacelike infinity the total gravitational energy is nonvanishing, whereas for these observers we have $\phi_{a b} \cong 0$ (in the limit $r \rightarrow \infty$; see next section).

\section{Static frames in the Kerr spacetime}

Another interesting application of the definitions of velocity and inertial acceleration of a frame discussed in section 3 is the analysis of a static frame in Kerr's spacetime. The latter is established by the line element

$$
\begin{aligned}
d s^{2}= & -\frac{\psi^{2}}{\rho^{2}} d t^{2}-\frac{2 \chi \sin ^{2} \theta}{\rho^{2}} d \phi d t+\frac{\rho^{2}}{\Delta} d r^{2} \\
& +\rho^{2} d \theta^{2}+\frac{\Sigma^{2} \sin ^{2} \theta}{\rho^{2}} d \phi^{2}
\end{aligned}
$$

with the following definitions:

$$
\Delta=r^{2}+a^{2}-2 m r,
$$




$$
\begin{aligned}
\rho^{2} & =r^{2}+a^{2} \cos ^{2} \theta, \\
\Sigma^{2} & =\left(r^{2}+a^{2}\right)^{2}-\Delta a^{2} \sin ^{2} \theta, \\
\psi^{2} & =\Delta-a^{2} \sin ^{2} \theta, \\
\chi & =2 a m r .
\end{aligned}
$$

A static reference frame in Kerr's spacetime is defined by the congruence of timelike curves $u^{\mu}(s)$ such that $u^{i}=0$, namely, the spatial velocity of the observers is zero with respect to static observers at spacelike infinity. Since we identify $u^{i}=e_{(0)}{ }^{i}$, a static reference frame is established by the condition

$$
e_{(0)}^{i}=0 \text {. }
$$

In view of the orthogonality of the tetrads, the equation above implies $e^{(k)}{ }_{0}=$ 0 . This latter equation remains satisfied after a local rotation of the frame, $\tilde{e}^{(k)}{ }_{0}=\Lambda^{(k)}{ }_{(j)} e^{(j)}{ }_{0}=0$. Therefore condition (35) determines the static character of the frame, up to an orientation of the frame in the three-dimensional space.

A simple form for the tetrad field that satisfies Eq. (35) (or, equivalently, $\left.e^{(k)}{ }_{0}=0\right)$ reads

$$
e_{a \mu}=\left(\begin{array}{cccc}
-A & 0 & 0 & -B \\
0 & C \sin \theta \cos \phi & \rho \cos \theta \cos \phi & -D \sin \theta \sin \phi \\
0 & C \sin \theta \sin \phi & \rho \cos \theta \sin \phi & D \sin \theta \cos \phi \\
0 & C \cos \theta & -\rho \sin \theta & 0
\end{array}\right),
$$

with the following definitions

$$
\begin{aligned}
A & =\frac{\psi}{\rho}, \\
B & =\frac{\chi \sin ^{2} \theta}{\rho \psi}, \\
C & =\frac{\rho}{\sqrt{\Delta}}, \\
D & =\frac{\Lambda}{\rho \psi} .
\end{aligned}
$$

In the expression of $D$ we have 


$$
\Lambda=\left(\psi^{2} \Sigma^{2}+\chi^{2} \sin ^{2} \theta\right)^{1 / 2} .
$$

We are interested in the calculation of $\phi_{a b}$ given by Eq. (17), and for this purpose it is useful to work with the inverse tetrad field $e_{a}{ }^{\mu}$. It reads

$$
e_{a}{ }^{\mu}=\left(\begin{array}{cccc}
\frac{\rho}{\psi} & \frac{\rho \chi}{\psi \Lambda} \sin \theta \sin \phi & -\frac{\rho \chi}{\psi \Lambda} \sin \theta \cos \phi & 0 \\
0 & \frac{\sqrt{\Delta}}{\rho} \sin \theta \cos \phi & \frac{\sqrt{\Delta}}{\rho} \sin \theta \sin \phi & \frac{\sqrt{\Delta}}{\rho} \cos \theta \\
0 & \frac{1}{\rho} \cos \theta \cos \phi & \frac{1}{\rho} \cos \theta \sin \phi & -\frac{1}{\rho} \sin \theta \\
0 & -\frac{\rho \psi}{\Lambda} \frac{\sin \phi}{\sin \theta} & \frac{\rho \psi}{\Lambda} \frac{\cos \phi}{\sin \theta} & 0
\end{array}\right),
$$

where now the index $a$ labels the columns, and $\mu$ the lines.

The frame determined by Eqs. (36) and (38) is valid in the region outside the ergosphere. The function $\psi^{2}=\Delta-a^{2} \sin ^{2} \theta$ vanishes over the external surface of the ergosphere (defined by $r=r^{\star}=m+\sqrt{m^{2}-a^{2} \cos ^{2} \theta}$; over this surface $g_{00}=0$ ), and we see that various components of Eqs. (36) and (38) are not well defined over this surface. It is well known that it is not possible to maintain static observers inside the ergosphere of the Kerr spacetime.

By inspecting Eq. (38) we see that for large values of $r$ we have

$$
e_{(3)}^{\mu}(t, r, \theta, \phi) \cong(0, \cos \theta,-(1 / r) \sin \theta, 0),
$$

or

$$
e_{(3)}^{\mu}(t, x, y, z) \cong(0,0,0,1) .
$$

Therefore we may assert that the frame given by Eq. (37) is characterized by the following properties: (i) the frame is static, because Eq. (35) is verified; (ii) the $e_{(3)}^{\mu}$ components are oriented along the symmetry axis of the black hole (the $z$ direction). The second condition is ultimately reponsible for the simple form of Eq. (36).

The evaluation of $\phi_{a b}$ is long but straightforward, and for this reason we will omit the details of the calculations. For convenience of notation we define the vectors

$$
\begin{aligned}
& \hat{\mathbf{r}}=\sin \theta \cos \phi \hat{\mathbf{x}}+\sin \theta \sin \phi \hat{\mathbf{y}}+\cos \theta \hat{\mathbf{z}} \\
& \hat{\theta}=\cos \theta \cos \phi \hat{\mathbf{x}}+\cos \theta \sin \phi \hat{\mathbf{y}}-\sin \theta \hat{\mathbf{z}}
\end{aligned}
$$


which have well defined meaning as unit vectors in the asymptotic limit $r \rightarrow \infty$. We also define the three-dimensional vectors

$$
\begin{aligned}
\mathbf{a} & =\left(\phi_{01}, \phi_{02}, \phi_{03}\right), \\
\boldsymbol{\Omega} & =\left(\phi_{23}, \phi_{31}, \phi_{12}\right) .
\end{aligned}
$$

We obtain the following expressions for $\mathbf{a}$ and $\boldsymbol{\Omega}$ :

$$
\begin{aligned}
\mathbf{a} & =\frac{m}{\psi^{2}}\left[\frac{\sqrt{\Delta}}{\rho}\left(\frac{2 r^{2}}{\rho^{2}}-1\right) \hat{\mathbf{r}}+\frac{2 r a^{2}}{\rho^{3}} \sin \theta \cos \theta \hat{\theta}\right] \\
\boldsymbol{\Omega} & =-\frac{\chi}{\Lambda \rho} \cos \theta \hat{\mathbf{r}}+\frac{\psi^{2} \sqrt{\Delta}}{2 \Lambda \rho} \sin \theta \partial_{r}\left(\frac{\chi}{\psi^{2}}\right) \hat{\theta}-\frac{\psi^{2}}{2 \Lambda \rho} \sin \theta \partial_{\theta}\left(\frac{\chi}{\psi^{2}}\right) \hat{\mathbf{r}} .
\end{aligned}
$$

The specific functional form of the vectors above completely characterize the frame determined by Eq. (36). The determination of $\mathbf{a}$ and $\boldsymbol{\Omega}$ is equivalent to the fixation of six components of the tetrad field. Equations (43) and (44) represent the inertial accelerations that one must exert on the frame in order to verify that (i) the frame is static (condition (35)), and that (ii) the $e_{(3)}{ }^{\mu}$ components of the tetrad field asymptotically coincides with the symmetry axis of the black hole.

The form of a and $\boldsymbol{\Omega}$ for large values of $r$ is very interesting. It is easy to verify that in the limit $r \rightarrow \infty$ we obtain

$$
\begin{aligned}
& \mathbf{a} \cong \frac{m}{r^{2}} \hat{\mathbf{r}} \\
& \mathbf{\Omega} \cong-\frac{a m}{r^{3}}(2 \cos \theta \hat{\mathbf{r}}+\sin \theta \hat{\theta}) .
\end{aligned}
$$

After the identifications $m \leftrightarrow q$ and $4 \pi m a \leftrightarrow \bar{m}$, where $q$ is the electric charge and $\bar{m}$ is the magnetic dipole moment, equations (45) and (46) resemble the electric field of a point charge and the magnetic field of a perfect dipole that points in the $z$ direction, respectively. These equations represent a manifestation of gravitoelectromagnetism.

If we abandon the statical condition given by Eq. (35), an observer located at a position $(r, \theta, \phi)$ will be subject to an acceleration $-\mathbf{a}$ and to a rotational motion determined by $-\boldsymbol{\Omega}=\boldsymbol{\Omega}_{D}$, which is the dragging frequency 
of the frame. Thus the gravitomagnetic effect is locally equivalent to inertial effects in a frame rotating with frequency $-\Omega_{D}$, the latter having the magnetic dipole moment structure given by Eq. (46). This is precisely the gravitational Larmor's theorem, discussed in Ref. [16].

The emergence of gravitoelectromagnetic (GEM) field quantities in the context of the acceleration tensor $\phi_{a b}$ presents no difference with respect to the usual approach in the literature. Let us assume that tetrad field satisfies the boundary conditions

$$
e^{a}{ }_{\mu} \cong \delta_{\mu}^{a}+\frac{1}{2} h_{\mu}^{a},
$$

where $h^{a}{ }_{\mu}$ is the perturbation of the flat space-time tetrad field in the limit $r \rightarrow \infty$, and that in this limit the $\mathrm{SO}(3,1)$ and spacetime indices acquire the same significance. It is straightforward to verify that in this case we have

$$
\begin{aligned}
\phi_{(0)(i)} \cong-\partial_{i}\left(\frac{1}{2} h_{00}\right)-\partial_{0}\left(-\frac{1}{2} h_{0 i}\right) \\
\phi_{(i)(j)} \cong-\left[\partial_{i}\left(-\frac{1}{2} h_{0 j}\right)-\partial_{j}\left(-\frac{1}{2} h_{0 i}\right)\right] .
\end{aligned}
$$

Thus we identify

$$
\begin{aligned}
V & =\frac{1}{2} h_{00} \\
A_{i} & =-\frac{1}{2} h_{0 i} .
\end{aligned}
$$

The identification above is equivalent to the one usually made in the literature, namely, $\Phi=(1 / 4) \bar{h}_{00}$ and $A_{i}=-(1 / 2) \bar{h}_{0 i}[17]$, where $\bar{h}_{\mu \nu}$ is the tracereversed field quantity defined by $\bar{h}_{\mu \nu}=h_{\mu \nu}-(1 / 2) \eta_{\mu \nu} h$, and $h=\eta^{\mu \nu} h_{\mu \nu}$. The latter identification is made directly in the weak field form of the metric tensor of a slowly rotating source. Assuming that $h_{00}=2 \Phi / c^{2}$ and $h_{i j}=\delta_{i j} h_{00}$, where $c$ is the speed of light (according to Eq. (1.4) of Ref. [17]), we obtain $\bar{h}_{00}=2 h_{00}$, and therefore $V=(1 / 4) \bar{h}_{00}$. To our knowledge, the identification of the GEM field quantities out of the tensor $\phi_{a b}$ has not been addressed in the literature so far. 


\section{Comments}

Gravity theories invariant under the global $\mathrm{SO}(3,1)$ group are physically acceptable. The gravitational field equations determine the gravitational field, not the frame. A given gravitational field configuration admits an infinity of frames which in general are distinct from each other. We know that the physical properties of a system are different in a static and in an accelerated frame, for instance, and this feature should also hold in general relativity.

The gravitational energy-momentum that is defined in the realm of the TEGR is frame dependent. This issue has been partially discussed before in Refs. [7, 8], and also in Ref. [9]. This dependence is considered here to be a natural property of the definition. The frame may be characterized by the six components of the antisymmetric tensor $\phi_{a b}$, defined by Eq. (17), which determine the translational acceleration and rotational frequency of the frame, and which resembles the electric field of a point charge and the magnetic field of a dipole, respectively, in the weak field limit of the Kerr spacetime (in the consideration of a static frame).

In section 4 we have shown that the gravitational energy-momentum calculated out of a frame that is nonrotating and freely falling in the Schwarzschild spacetime vanishes. We expect this property to hold in the consideration of a general spacetime geometry, in which case the analysis is somewhat more

complicated, because the frame is expected not to rotate with respect to a Fermi-Walker transported frame. In general the construction of the latter frame is not trivial.

It is clear that if the gravitational energy-momentum definition were invariant under local Lorentz transformations, we would not arrive at the result of section 4 , since the the value of $P^{a}$ on a three-dimensional volume $V$ would be the same for all frames, and presumably nonvanishing.

A common critique of the localizability of gravitational energy is that the latter is unattainable because of the principle of equivalence. In this paper we have seen that this is not the case. Definition (7) for the gravitational energy-

momentum yields the expected results both for observers asymptotically at rest and for freely falling observers.

\section{Acknowledgement}

J. W. M. is grateful to G. F. Rubilar for helpful discussions on reference frames. This work was supported in part by CNPQ (Brazil). 


\section{References}

[1] F. W. Hehl, J. D. McCrea, E. W. Mielke and Y. Ne'eman, Phys. Rep. 258, 1 (1995).

[2] F. W. Hehl, in "Proceedings of the 6th School of Cosmology and Gravitation on Spin, Torsion, Rotation and Supergravity", Erice, 1979, edited by P. G. Bergmann and V. de Sabbata (Plenum, New York, 1980).

[3] J. M. Nester, Int. J. Mod. Phys. A 4, 1755 (1989); J. Math. Phys. 33, 910 (1992).

[4] J. W. Maluf, J. F. da Rocha-Neto, T. M. L. Toríbio and K. H. CastelloBranco, Phys. Rev. D 65, 124001 (2002).

[5] J. W. Maluf, F. F. Faria and K. H. Castello-Branco, Class. Quantum Grav. 20, 4683 (2003).

[6] Y. Obukhov and J. G. Pereira, Phys. Rev. D 67, 044016 (2003).

[7] J. W. Maluf, Ann. Phys. (Leipzig) 14, 723 (2005).

[8] J. W. Maluf, S. C. Ulhoa, F. F. Faria and J. F. da Rocha-Neto, Class. Quantum Grav. 23, 6245 (2006).

[9] Y. N. Obukhov and G. F. Rubilar, Phys. Rev. D 73, 124017 (2006).

[10] V. C. de Andrade and J. G. Pereira, Phys. Rev. D 56, 4689 (1997).

[11] J. W. Maluf and J. F. da Rocha-Neto, Phys. Rev. D 64, 084014 (2001).

[12] F. H. Hehl, J. Lemke and E. W. Mielke, Two Lectures on Fermions and Gravity, in "Geometry and Theoretical Physics", edited by J. Debrus and A. C. Hirshfeld (Springer, Berlin Heidelberg, 1991).

[13] B. Mashhoon and U. Muench, Ann. Phys. (Leipzig) 11, 532 (2002) [grqc/0206082].

[14] B. Mashhoon, Ann. Phys. (Leipzig) 12, 586 (2003) [hep-th/0309124].

[15] J. B. Hartle, "Gravity: An Introduction to Einstein's General Relativity" (Addison-Wesley, San Francisco, 2003), p. 198. 
[16] B. Mashhoon, Phys. Lett. A 173, 347 (1993).

[17] B. Mashhoon, Gravitoelectromagnetism: a Brief Review [gr-qc/0311030]. 